same time and in the same quantity, as in two pea-seeds grown under similar circumstances.

Similarity of growth may be partial ; in two subjects the growth may be similar as to time, or quantity, or kind. When two processes of growth are different in kind, they may be similar in time; the growth of leaves and of underground tubers may occur at the same time, and this may be the only point of similarity between the two processes of growth. (Illustrative examples were given.)

In classifying facts concerning the processes of growth, according to such intrinsic attributes as are observable, our object is to seek the causation of the attributes observed. Having classed a number of facts as examples of unequal bilateral growth, we may seek to determine the causation of unequal bilateral growth. In pursuing such methods of inquiry, we follow methods similar to those which aided us in studying the motor functions of the brain. Thus we have lines of thought to guide observation. It is possible, and, I think, highly desirable, to arrange a museum in which specimens are classified according to the attributes of their growth and the forces that stimulated their growth and produced that special ratio of their attributes. It seems to me that this was in part the principle followed by John Hunter.

A catalogue was shown in which specimens were thus arranged.

The rules that should be followed in making analogies were pointed out. It was shown that we can only compare things or acts as to the characters or attributes which they possess in common ; we may com. pare like functions as to their time and quantity ; it is only as to the time of action that we can compare unlike functions; we do not directly compare the material things, but the attributes of their functions. Growth in a child and in a plant were then compared, and other examples were put forward.

In our desire to give some kind of explanation of facts in Nature, we used processes of analogy. Acceptance of the hypothesis of evolution encourages the study of analogies; our purpose in making analogies between processes of growth is to classify examples in the best manner for seeking their causation.

The term "reversion" is used to imply that the outcome of growth observed is similar in its intrinsic attributes to others that have previously been observed. The reversion is a repetition or reappearance of vital acts, similar in their intrinsic attributes and occurring in similar subjects.

The following proposition was enunciated as an epitome of the principles illustrated : "Every vital act, or act of growth, requires among its antecedents a supply of pabulum and stimulation by some force incident to the subject."

\section{NOTE ON PIANISTS' CRAMP.}

\section{BY WARRINGTON HAWARD, F.R.C.S., Surgeon to St. George's Hospital.}

I HAVE read with much interest Dr. Vivian Poore's lecture in the JoURNAL of February 26 th on certain conditions of the hand and arm which interfere with piano-playing. It seems to me that cases of functional failure in pianists have become much more common in recent years ; and as it is convenient to have some name for the condition, probably that of "pianists' cramp" "which has already been applied to it, is sufficiently characteristic, and has the advantage of suggesting its alliance with the well-known affection, "writers" cramp."

No doubt, as Dr. Poore says, failure in piano-playing " may depend upon a variety of causes," and he alludes to the effect of gouty, rheumatic, and other changes in the disabled members. But the condi. tion for which the name of pianists' cramp may be used is quite peculiar, and is always the result of over-fatigue of the affected part. Of course, "over-fatigue" is a relative term. An amount of pianoplaying that can be practised without harm by one pupil will over. fatigue another; and in the dyspeptic, anæmic, and ill-nourished the muscles will break down under less prolonged use than in the healthy. But in the majority of cases of failure of the muscles of piano-playing, which occur without obvious disease or injury, overuse of the muscles will be found to be the essential cause. A potent predisposing cause is very frequently to be found in the anæmia and general ill-nourishment of the body which result from too much confinement within doors, and the accompanying dyspepsia and lack of appetite ; and this has, in several instances within my knowledge, been aggravated by the change in diet, climate, and other habits of life involved in leaving an English home for residence and study on the Continent.

My experience of these cases is much less than Dr. Poore's, and all that I have seen have been young people, during or just emerging from the period of pupilage. In one rather severe case, accompanied by much muscular aching, the little and ring fingers of the left hand became, on any attempt to play, cold, benumbed, and purple ; and these, I think, are the fingers specially liable to give way. I have recently seen an instance of a precisely similar condition affecting the little and ring-fingers of the right hand, with pain in the flexor muscles, in a rather delicate young lady given to much and rapid knitting.

Of the treatment of this condition, doubtless, as Dr. Poore points out, the most important part is rest and attention to the general nutrition of the body. Careful maintenance of the warmth of the affected limb (which is usually cold) has also seemed to me to be helpful; and I am sure that I have seen marked benefit from moderate and intelligently applied massage, even in cases where there has been nerve-tenderness ; in two cases the pain always being relieved thereby. I think that, at least in some instances, the muscular tension involved in what Dr. Poore has well called the "steadying" of the limb for piano-playing, produces a considerable amount of venous congestion; and it is noticeable that long practising is apt. to make the hands cold.

I am glad to see that Dr. Poore does not approve of the division of the fibrous bands which connect the extensor tendons of the ringfinger to the tendons on either side of it, which has been proposed as a means of giving greater freedom to the ring-finger of pianists. Can. anyone suppose that the delicacy or power of touch of any of our great pianists would "be improved by this absurd and unscientific proceeding ?

\section{TINNITUS AURIUM, AND ITS TREATMENT BY A NEW METHOD OF ALTERNATE INJECTION AND EVACUATION OF AIR. ${ }^{1}$}

BY JOHN WARD COUSINS, M.D.LOND., F.R.C.S., Senior Surgeon to the Royal Portsmouth Hospital, and to the Portsmouth and South Hants Eye and Ear Infirmary.

[Concluded from page 44\%.]

A FEW words now with reference to prognosis. It is not always an easy matter to express a confident opinion at once as to the complete or only partial restoration of the organ. In favourable cases the intensity of the aural symptoms often undergoes great variation from. time to time. As a general rule, whenever the hearing power improves, the tinnitus is less disturbing; so that these symptoms appear to rise and fall alternately, and under very slight differences of tympanic pressure. Tinnitus, when dependent solely upon middleear disease, may be regarded as a curable disturbance; and, even in chronic cases, the relief obtained is often very considerable, and bears a direct relation to the amount of hearing power regained. On the other hand, the issue is always doubtful and unsatisfactory whenever there is repeated evidence of a deficiency in the cranial conduction of sound, especially when this loss of conducting power has been long associated with gradually increasing deafness and tinnitus. There is frequently, too, a marked relation between the degree of deafness, and the unvarying character of the aural confusion; and, in some cases of total and hopeless loss of hearing power, the persistent tinnitus forms the most distressing part of the disorder.

Let us now briefly consider the question of treatment. Many in. ternal remedies have been recommended for this aural affection, but. I have very little confidence in any of them, as in my hands they have done very little good, even when administered for a considerable period. The fact is, we know of no specific remedies for tinnitus aurium. The removal of this symptom wholly depends upon the successful treatment of the special morbid process by which it is excited. If we can succeed in curing the aural disease, and in restoring the integrity of the organ as a sound-receiving instrument, all the concomitant disorders will at once disappear.

With these preliminary remarks, I shall proceed at once to the treatment of tinnitus dependent upon disease of the middle ear; and this, permit me to remind you, is the special topic of my address. Theoretically, the treatment of this form of tinnitus is a very easy matter, and simply consists in promoting free and healthy communication between the tympanum and the pharynx, and so restoring the normal balance of pressure on both sides of the membrana tympani. If pent-up fluid exists in the cavity, it must be evacuated, and its mucous surface stimulated to healthy eecretion. If chronic changes are present, producing collapse of the drum and immobility of the bony chain, our efforts must be directed to promote the normal elasticity of these parts, and, if possible, to re-establish their intrinsic

3 Address delivered before the South-East Hants Medical Society. 
movements. On the other hand, if the Eustachian tubes are ob. structed by secretion, our remedies must be employed for the purpose of clearing the canals, and restoring the normal condition of the naso-pharynx and throat.

The application of the air-douche has long been recognised as an invaluable method of treatment, and it is especially serviceable in the early stages of middle-ear disease. But in chronic cases, attended by obstinate tinnitus, it tends to aggravate the tympanic pressure. It often succeeds in opening up the tubes, but the simple injection of air is followed by a distressing sensation of distension, and a temporary increase of all the symptoms. The disturbance, however, generally soon subsides, as the imprisoned air gets rapidly absorbed. When the cavity is filled with mucus, the air-douche increases still more the abnormal pressure, and fails to dislodge the pent-up secretion, so that the membrana tympani becomes unduly and dangerously tense, and, in this condition, accidents have occasionally occurred.

The treatment which I have practised during the past two years, with very great succees, consists essentially in the injection of air, and its immediate evacuation. Sometimes the air is medicated by the vapour of some volatile substance, for the purpose of reducing the sensibility of the cavity and stimulating the mucous surface. In many cases the operation removes at once the abnormal pressure, and yields immediate relief. It is a simple modification of the ordinary air-douche; but, at the same time, I maintain that it renders this method far more efficient in a large number of cases of middle-ear disease, and removes the risk of causing aural discomfort, and of increasing tympanic tension.

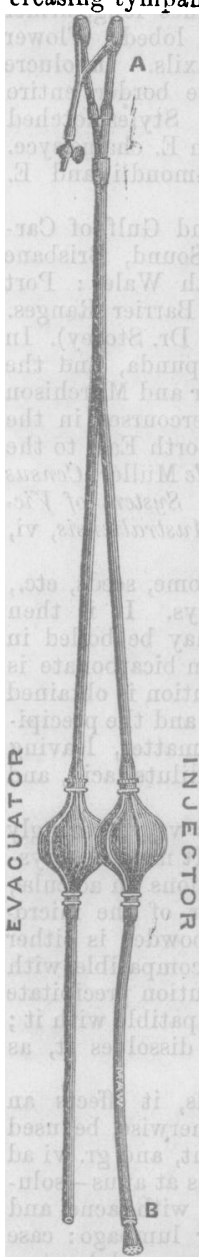

The apparatus which I employ for this operation is represented in the engraving. It consists of two parts : 1. A special nasal piece for hermetically closing the nose. 2. Two powerful hand-balls, which are connected with the former by an india-rubber tube. The nasal piece is composed of two tubes, with their extremities enclosed in inflatable bags made of very thin and soft rubber. The inflating tube has a separate connection with the lower part of each bag. The hand-balls are fitted with recoil springs, which impart force and rapidity to their expansion. One hand-ball is marked for injection, the other for evacuation, and their converse action is obtained by simply reversing the valvular arrangement, so that the outlet valve is fixed to the connecting tube on one side, and the iulet valve on the other.

When medicated air is to be injected, the selected fluid must be dropped upon a pledget of cotton-wool placed in the conical vulcanite receptacle (B), which is attached to the end of the injecting-tube. By the action of the hand-ball the air is drawn through the perforated lid, and thus charged with vapour. In this way I often employ chloric ether, chloroform, creasote, iodine, carbolic acid, iodoform and ether, either alone or in some combination.

The operation of alternate injection and evacuation of the tympanum is performed in the following manner. The nasal orifices are, first of all, carefully wiped dry with cotton $\cdot$ wool, the nasal piece is placed in position, and the bags are inflated so as hermetically to close the nose. The patient, supporting the vulcanite stem between the left forefinger and thumb, is directed to take a full inspiration, and then, while holding firmly the breath and pressing the lips together, to perform a muscular movement, just as if he were in the act of blowing through a tube. The operator now compresses the evacuating-hall with the left hand, and with the other hand-ball he injects air by two or three contractions of the right hand. The left hand is then suddenly relaxed, and the evacuator expands by the force of its elasticity, aided by the recoil-spring within it. After three or four of these movements, which with a little practice can be done with great rapidity, the patient is requested to breathe again, and then to take another full inspiration. In this way the injection and evacuation of air can be repeated several times in succession at one sitting. The hearing power should always be tested with the watch, both before and after each operation; and, during its performance, the otoscope can be employed with advantage, but the operator may generally rely upon the intelligent observation of the patient regard- ing the movements of the drum, and the entrance of air into the tympanic cavity.

The alternate method which I have described presents many advantages over other forms of tubal inflation. It promotes the imme. diate discharge of pent-up secretion into the pharynx, and aids in establishing the drainage of the tympanum. By the injection of air, the mucus is disturbed from its position, and by reversing the action it can be drawn towards the tube, and its passage to the throat greatly accelerated. It proves also of material assistance in the treatment of collapsed and adherent membrane. In chronic cases the mobility of the bony chain has been long impaired by the morbid condition of the middle ear ; but, by gentle and repeated agitation in both directions, the adhesions between the ossicles are loosened and their normal oscillation restored, so that sonorous vibrations can be again transmitted from the drum to the fluid within the labyrinth.

With reference to the repetition of the operation, in every case we must simply be guided by results. In recent cases, very considerable relief is often obtained at one sitting, and patients put off another operation until they get a recurrence of their aural trouble. Many of my patients derive so much benefit from the treatment that they return again and again at irregular intervals to have it repeated. In chronic cases, however, improvement is generally slow, and the operation has to be perseveringly continued for several weeks. It is my usual plan to practise it two or three times every week, and to excite the to-and-fro movement of the membrans tympani freely at each sitting. With intelligent patients, especially those residing at a distance, I often teach them to perform the operation gently every day with my simple aural inflator, a description of which has already appeared in this JourNaL. ${ }^{2}$

Now, it would be very easy for me to relate many instances of success in cases of obstinate tinnitus aurium, even after the persistent trial of other forms of treatment ; but it will be quite sufficient for my purpose if I briefly mention two or three examples, with the hope that my experience in a large number of similar cases may induce some of my friends to test and criticise my treatment.

CASE I. - E. S., an officer in the Royal Navy, had suffered for some months from deafness and aural confusion. The attack followed from exposure after syringing the ears for the removal of impacted wax. The tinnitus was often intolerable, and he said that it would drive him mad if it continued. He had tried blistering, the air-douche, and other remedies. The operation of alternate injection and evacuation was performed three times a week for one month, and then only occasionally. He expressed great relief at each sitting, and in three months he completely recovered normal hearing power, and lost all the distressing symptoms caused by tension within the tympanum.

CASE II.-M. M., a married lady, consulted me, about eighteen months ago, for deafness on the left side and distracting tinnitus. She had been treated by several surgeons, and had been recommended to use the ordinary Politzer's bag; but this always increased her trouble. I at once commenced the treatment of alternate injection and evacuation, and directed an anodyne and stimulating liniment to be rubbed behind the ear. The operation was repeated several times with permanent binefit.

CASE III.-F. S., a commercial man, had suffered from deafness and noises in the head for twelve months. The symptoms had been very variable, and an aggravation of the tinnitus occurred whenever his hearing power was much disturbed. In all other respects he appeared a healthy man, and the disorder was wholly dependent upon aural mischief. He had been blistered freely, and bad taken a great deal of medicine. After three weeks' treatment the deafness cleared away, and he completely lost the tinnitus, which had constantly distressed him for many months.

In acute disorders of the middle ear, the operation is also often of great value. The tinnitus and pain which attend catarrhal inflammation are immediately relieved by the double action of my instrument. By its gentle application the tension caused by increased secretion is removed, and the fluid escapes into the pharynx. Of course other local remedies, such as hot fomentations and leeehes, are useful in these cases, together with general treatment by purgative and sedative medicines. In acute suppuration of the middle ear, with bulging of the tympanic membrane, evacuation in this way may sometimes succeed, preventing the rupture of the drum, and obviating the necessity of puncture. But in the severe forms of suppurative otitis which follow scarlet fever and other zymotic diseases, or which occur in connection with long-standing aural mischief, there must be no delay ; for it is the bounden duty of the surgeon to be an eye-wit.

2 October 3rd, 1885 (page 641). Messrs. Maw, Son and Thompson are the makers of my aural instruments. 
ness to the evacuation of the pent-up pus, and to rely only on the timely performance of paracentesis.

I must now refer to several matters which interfere with the easy and successful performance of my operation at the onset. Some patients are timid and excitable, and, under these circumstances, it often requires a little tact to gain their confidence and obtain their voluntary assistance. Another difficulty arises from the imperfect closure of the nostrils. For the successful application of the apparatus, it is absolutely necessary to adjust the inflatable nose-picce, so that the orifices are securely and completely plugged. I have at hand several nasal tubes, and it is always easy to select one 'to suit the nostrils of the patient. Again, it is equally essential to attend to the closure of the lips, and the fixing of the muscles of the chest and pharynx. After taking the full inspiration, the patient must perform the muscular movement of forced expiration, without permitting the discharge of air by the mouth. In this way, by the action of the tensors of the palate and the muscular structures of the pharynx, the cartilaginous orifices of the Eustachian tubes are drawn downwards, and rendered patent, so that air can readily pass in and out of the tympanum. The muscular movements which take place during deglutition act directly upon the open ends of the tubes; and, for this reason, it is often very useful, during the early stages of the operation, to get the patient to swallow a little water several times at regular intervals.

Another cause of delay and difficulty arises from those chronic dis. orders of the throat and naso-pharynx which are so intimately associated with middle-ear disease. A relaxed and congested condition of these parts must be treated by the persistent application of local astringents, applied regularly and freely several times during the day. If the tonsils are enlarged and indurated by inflammatory changes, the ordinary operation of excision will be necessary. When a thick muco-purulent secretion from the pharyngeal follicles occludes the Eustachian orifices, it requires to be constantly washed away, and the mucous surface of the cavities stimulated by deodorising solutions. These applications can be very efficiently made by the patient himself, and he should be instructed to introduce them into the nostrils with a syringe, and then to draw them over the back part of the velum palati into the mouth, and thus dislodge the mucus.

Sometimes the naso-pharynx is blocked by adenoid growths of very variable consistency. These vegetations seriously obstruct the tubal openings, and cause obstinate deafness, with derangement of tympanic pressure. Now, before my method is applied for the relief of the aural symptoms, it is essential that these mucous outgrowths should be removed. A very effectual plan is to explore the pharynx thoroughly with the forefinger; and then, wherever vegetations are detected, to serape them off at once with the nail. I have performed this little operation for some years, at the suggestion of Mr. Cresswell Baber, of Brighton, with excellent results. Sometimes it excites so much struggling and retching that the administration of an anesthetic is necessary for its successful performance. Sir William Dalby has recently introduced a steel nail for the removal of these pharyngeal growths. It is secured over the end of the finger without covering the point; and by its aid, he states, the cavities can be cleared with great facility at one sitting.

In many cases of chronic and complex aural disease, in which tin. nitus is dependent upon disorder of both the nervous and conducting structures of the organ, very considerable benefit is obtained by the operation of alternate injection and evacuation. It is especially, too, in these cases that the induced current proves of much value as an auxiliary measure. It acts directly as a stimulant to the nervous and muscular structures. The morbid changes in the tympanum have been followed by wasting and paralysis of the intrinsic muscles; and the tinnitus, often of a very depressing and continuous character, is occasionally complicated with attacks of aural vertigo. I always em. ploy a weak current, and conduct it directly on to the tympanic membrane, near its posterior border, by means of a loop of silver wire enclosed in a vulcanite speculum. The electrical stimulant generally excites some contractions in the facial muscles, and sometimes seems to increase for a time the sensibility of the auditory nerve. By repeated and judicious application, relief is often obtained; and this result can be fairly attributed to increased tonicity of the tympanic muscles and a reduction of the abnormal tension upon the nervous apparatus of the organ.

In conclusion, many cases of tinnitus are undoubtedly aggravated by morbid conditions of the blood, and diseases of the general system. When ansemia, gout, syphilis, or rheumatism are present, appropriate remedies must be employed, and patients must be strictly enjoined to live regularly and temperately.

As regards the treatment of tinnitus in cases of severe and hopeless deafness caused by disease of the deep recesses of the labyrinth, some benefit may be obtained by the application of the induced current, and by the use of stimulating applications behind the ears. The injection of air into the middle ear containing a little of the vapour of either chloroform, ether, or carbolic acid, is also serviceable, and can be advantageously employed by the patient himself whenever the symptoms are urgent. But respecting the treatment of these obscure aural orders, I have really no suggestion to offer. A combination of deafness and tinnitus, produced by abnormal tension within the tympanum, is the most common of all aural derangernents; and I can confidently recommend the alternate injection and evacuation of air as an improvement upon the local measures which are at present adopted for restoring the normal equilibrium of pressure within the tympanic cavity.

\section{DRUMINE.}

By JOHN REID, M.A., M.D., C.M.GLAs., Melbourne, Australia.

DRUMINE is an alkaloid obtained from Euphorbia drummondii (Boiss). Euphorbia drummondii, N.O. Euphorbiaceæ, has the following botanical characters. Prostrate or diffuse much-branched plant, closely resembling the European E. chamæsyce (Linn.), and when flowering the first year easily confounded with it; but always quite glabrous, and forming at length perennial thick or woody rhizome. Leaves opposite, orbicular ovate or oblong, obtuse or notched, entire or serrulate, very oblique, at the base two to four lines long, firmer than in E. chamæsyce. Stipules entire fringed or lobed. Flower heads very small, shortly pedicellate in the upper axils. Involucre about half a line long, the glands with a narrow white border, entire or nearly so. Capsule under one line long, glabrous. Styles notched only, varying from almost none to nearly as long as in E. chamresyce. Seeds rugose, E. chamæsyce, E. Ferdudi, E. Drummondii and E. Dallachyana (Baill. Adans, vi, 284-85).

Habitat. - In North Australia : Victoria River and Gulf of Car. pentaria. In Queensland: Broad Sound, Thirsty Sound, Brisbane River, Rockhampton, Curriwillighie. In New South Wales: Port Jackson, Lachlan River, Darling River, thence to Barrier Ranges. In New Kealand. In Tasmania : Swanport (found by Dr. Storey). In South Australia: Spencer's Gulf, Murray River, Kapunda, and the Gatoler Ranges. In Western Australia: Swan River and Murchison River. In Victoria: From the sources of the watercourses in the North-West to the Murray River, from those in the North East to the Hume River, including the whole Victorian Alps. Vide Miiller, Census of Australiun Plants Fraymenta, vi, p. $182 ; K e y$ to $S^{\prime} y$ stem of $Y^{r} i c$ torian Plants, 1885, p. 11, and Bentham's Florc Australiensis, vi, p. 49.

Preparation. - The whole plant, including the rhizome, seeds, etc., is macerated in dilute hydrochloric acid for some days. It is then strained through linen under pressure. (The plant may be boiled in the dilute acid, and strained under pressure.) Sodium bicarbonate is added, stirring while adding it, until an alkaline solution is obtained with precipitated drumine. The product is filtered, and the precipitate is washed with water to get rid of colouring matter, leaving drumine on the filter. This may be dissolved in dilute acid, and evaporated to obtain the hydrochlorate, or other salt.

Properties. - Alkalies precipitate it, the salicylate is very sparingly soluble in water and gives a crystalline precipitate. It may be crystallised from aqueous, spirituous, or chloroform solutions in acicula, the larger ones almost rhombic, under a high power of the microscope, and stellate crystals, all microscopic. The powder is either dirty like morphine, or pure white. The solution is compatible with iron and acids, cyanide of potassium, and a neutral solution precipitate it, not prussic acid; perchloride of mercury is compatible with it ; strong nitric acid does not act on it chemically, but dissolves it, as does $\mathrm{H}_{2} \mathrm{~S} \mathrm{O}_{4}$

Uses. - In nasal catarrh, injected into the nostrils, it effects an immediate cure. This may, in chronic cases or otherwise, be used in solution with gr. $i$ corrosive sublimate to the pint, and gr. vi ad z i hydrochlorate of drumine. Spasmodic contractures at anus-solution locally; the same in pruritus. Facial dermatitis, with acne and itching: spermaceti $\tilde{z} i$, drumine gr. $x$. Sciatica or lumbago: case of two years' duration, unaffected by other remedies, cured by two injections. Sprains :-Cases of sprained abdominal muscles cured speedily by free injections. Periosteal pain following fracture of the lower end of the sternum, with tenderness, of two years' duration, cured by injections. Burns :- Extensive denudation of skin; very free application to raw surfaces; relief of pain; no constitutional ymptoms. Neuralgia:-Dropped into the eye, it gave relief. Opera. 